\title{
Effect of the pre-photorefractive keratectomy Schirmer value on the post-photorefractive keratectomy central epithelial thickness, ocular surface disease index scores, and uncorrected visual acuity
}

\section{Metin Ekinci}

Istinye University: Istinye Universitesi

İfan Botan Güneş ( $\square$ irfanoft@gmail.com )

Kocaeli Health and Technology University: Kocaeli Saglik ve Teknoloji Universitesi

https://orcid.org/0000-0002-4515-2310

\section{Hakan Öztürk}

Izmir Tepecik Egitim ve Arastirma Hastanesi: Izmir II Saglik Mudurlugu Izmir Saglik Bilimleri Universitesi Tepecik Egitim ve Arastirma Hastanesi

\section{Research Article}

Keywords: corneal epithelial thickness, dry eye, photorefractive keratectomy, visual acuity

Posted Date: February 4th, 2022

DOI: https://doi.org/10.21203/rs.3.rs-1217825/v1

License: (c) (1) This work is licensed under a Creative Commons Attribution 4.0 International License. Read Full License 


\section{Abstract}

Purpose: The aim of the present study was to investigate the effect of the pre-photorefractive keratectomy (PRK) Schirmer value on the post-PRK central epithelial thickness (CET), Ocular surface disease index (OSDI) scores and uncorrected visual acuity (UCVA).

Methods: After the exclusion of patients, 106 patients were analyzed ( $n=54$ for control group; $n=52$ for dry eye group). Best corrected visual acuity levels, detailed anterior segment and fundus examinations of the patients, intraocular pressures were recorded. Corneal thickness measurements were performed. Tear film break-up time (BUT) and Schirmer's tests were applied to all patients. The OSDI questionnaire was administered to all patients at the third month after the operation. The patients were divided into two groups according to the preoperative Schirmer's value as follows: control group (preoperative Schirmer value over $10 \mathrm{~mm}$ ) and dry eye group (preoperative Schirmer 1 value between $0-10 \mathrm{~mm}$ ). After the surgical procedure, the measurements and questionnaire were repeated.

Results: The mean OSDI score and CET value increased significantly in dry eye group at the third month after the operation compared to the preoperative period $(\mathrm{P}<.001)$. After PRK, UCVA does not show a significant difference between groups.

Conclusion: Laser surgery affects the corneal epithelial thickness in some patients and reduces visual comfort. Thus, it can be suggested that dry eye should be treated preoperatively, followed by PRK.

\section{Introduction}

Dry eye syndrome (DES) is a multifactorial common disease characterized by unstable tear film [1]. Although there is no exact cure for DES, the symptoms accompanied by DES can be managed by a variety of applications [2]. DES was classified as tear-deficient and evaporative, and further sub-classified as having intrinsic and extrinsic etiological factors [3]. It has been demonstrated that the prevalence of DES in women is higher than in men, and the incidence increases with age [4-5]. Apart from demographic properties, DES is one of the most common complications after refractive corneal surgery.

The aim of refractive corneal surgery is to increase or decrease refractive power by changing the structure of the cornea. A laser was first used on the human eye for refractive surgery by Seiller in 1990 [6]. Excimer lasers break down molecular organic bonds in sensitive structures, such as epithelial tissue and collagen, via photochemical reaction. With the ablative effect, the desired shape change is achieved in the cornea.

Photorefractive keratectomy (PRK) can cause injury to the epithelium, nerves, and upper stromal layers [7]. Despite the pharmacological drugs after PRK, patients can report postoperative pain or discomfort [8]. Although some clinical conditions are more painful, the pain reported by patients with similar clinical conditions shows high variance [9]. In order to provide effective analgesic treatment strategies, it is crucial to preoperatively distinguish patients who are expected to have high postoperative pain from lowrisk patients [10-11]. Some predictors, such as gender, anxiety, and knowledge of the procedure, have 
been previously reported for pain [12-14]. As for PRK, preoperative predictors of pain, such as gender, anxiety, knowledge of the procedure, and spherical equivalent refractive error, were previously reported [11].

The ocular surface disease index (OSDI) is a questionnaire aimed at questioning patients' complaints about ocular surface diseases. The OSDI questionnaire also scores the severity and duration of these complaints and their impact on daily activities [15].

The aim of the present study was to investigate the effect of the pre-PRK Schirmer test value on the postPRK central epithelial thickness (CET), OSDI scores, and uncorrected visual acuity. The null hypothesis was that no differences in the post-PRK CET, OSDI scores, and uncorrected visual acuity existed between the groups.

\section{Materials And Methods}

\section{Design}

The present study was a retrospective cohort study; the inclusion criteria were as follows: 1) patients who underwent PRK in their right eye due to myopia and 2) complete data. The exclusion criteria consisted of patients with allergic conjunctivitis; corneal pathology; glaucoma; those who wear contact lenses; systemic disease, such as diabetes; connective tissue disease; chronic topical or systemic drug use; and a history of ocular surgery.

The study consisted of 148 patients who underwent PRK in their right eye due to myopia between July 2017 and August 2020 in the ophthalmology clinic. After the exclusion of patients, 106 patients were analyzed ( $n=54$ for the control group; $n=52$ for the dry eye group). The present study was approved by the Istinye University Ethics Committee (2/2021.K-76). Informed consent was obtained from all subjects included in the study, and the standards of the Helsinki Declaration were followed.

\section{Clinical Procedures}

The detailed drug and medical histories of all the included patients were taken. Best corrected visual acuity levels (logMAR) were checked, and detailed anterior segment and fundus examinations of the patients were performed with a slit-lamp biomicroscope and a 90-diopter lens. Intraocular pressures were measured by non-contact tonometry (Topcon non-contact tonometer CT-11P, Tokyo, Japan). Manifest and cycloplegic refraction measurements were made (Topcon KR1 Auto-Kerato-Refractometer, Tokyo, Japan). Tear film break-up time (BUT) and Schirmer's tests were applied to all patients.

The Schirmer and BUT tests were performed three days before the operation and three months after the operation, without applying topical anesthesia. For the Schirmer test, readings were reported in millimeters of wetting for 5 minutes. For the BUT test, fluorescein dyed paper was contacted with the 
lower conjunctival fornix and waited until the fluorescein was completely dispersed on the corneal surface. The time from the last blink to the first break of the tear film was recorded in seconds.

The OSDI questionnaire was administered to all patients at the third month after the operation. The total OSDI score was calculated with the formula OSDI = ([sum of the scores of all questions answered] $x 25]$ / ([total number of questions answered]). The results were evaluated in the range of $0-100$ [16].

Corneal epithelial thickness maps of the patients were obtained before the operation and at the third month after the operation by using the corneal acquisition mode of the Spectral Domain Optical Coherence Tomography (SD-OCT) device (Optopol Technology sp.zo. Zawiercie Polska). The thickness values of the corneal epithelium were examined in a circular area with a diameter of $4 \mathrm{~mm}$. The corneal epithelial thickness value of the central area with a diameter of $2 \mathrm{~mm}$ was defined as the central corneal epithelial thickness (CET) (Figure 1).

\section{Groups}

The patients were divided into two groups according to the preoperative Schirmer test value, as follows

\section{Control group}

Preoperative Schirmer value over $10 \mathrm{~mm}$, and

\section{Dry eye group}

Preoperative Schirmer 1 value between $0-10 \mathrm{~mm}$.

\section{Surgical Method}

All surgical procedures were performed with the VISX Vue tm STAR S4 IR tm Excimer Laser by the one surgeon. Before the surgical procedure, the patients' eyelids were cleaned with $5 \%$ betadine solution. After their eyes were closed with a drape, topical anesthesia with proparacaine was applied. After applying $10 \%$ alcohol solution for 15 seconds with an $8 \mathrm{~mm}$ diameter corneal trephine, the corneal epithelium was debrided with a triangular sponge. After the corneal epithelium was debrided, stromal ablation was performed with a laser at a frequency of $400 \mathrm{~Hz}$ and a wavelength of $193 \mathrm{~nm}$ in the $6.5 \mathrm{~mm}$ ablation zone. Then, a $0.02 \%$ Mitomycin C soaked sponge was applied to the ablation site for 5 seconds. After washing with BSS solution, bandage contact lenses were applied to the eye and the operation was terminated.

In the postoperative period, moxifloxacin drops were used four times a day for one week, dexamethasone drops six times a day for one month, and preservative-free artificial tear drops (Artelac Advanced, Bausch \& Lomb, France) were used every two hours for a month. The patients were examined on the third day, tenth day, first month, and third month postoperatively. When corneal epithelization was complete, the bandage contact lenses were removed. 


\section{Statistical Analysis}

All statistical analyses were performed using SPSS ${ }^{\circledR}$ version 15 software (IBM SPSS Inc., Chicago, IL, USA) at a $95 \%$ confidence interval $(P=0.05)$. Multinomial logistic regression was used to predict a nominal dependent variable given independent variables. After the normality test, independent/paired samples t-tests (for homogeneous results) and Mann-Whitney $U$ tests (for non-homogenous results) were performed. For nominal data, the chi-square test was performed.

\section{Results}

The study included 52 eyes for the dry eye group and 54 eyes for the control group. The patients' demographic data is shown in Table 1; there were no statistically significant differences between the control group and the dry eye group in terms of demographic data (age and gender) $(P>0.05)$. Ablation depth, uncorrected visual acuity, and best corrected visual acuity levels were similar between the groups $(P>0.05)$ (Table 2). The pre/postoperative Schirmer test, BUT, OSDI, central epithelial thickness, and spherical equivalent refractive error, according to the groups, can be seen in Table 2.

It was found that the mean spherical equivalent refractive error and Schirmer test values decreased in the control group at the third month after the operation compared to the preoperative period $(P<.05)$. The mean BUT, OSDI score, and central epithelial thickness were similar in the control group at the third month after the operation and at the preoperative period $(P>.05)$ (Table 3$)$.

The mean ocular surface disease index score and the central epithelial thickness value increased significantly in the dry eye group at the third month after the operation compared to the preoperative period $(P<.001)$. However, the mean spherical equivalent refractive error and the Schirmer test values decreased in the dry eye group at the third month after the operation compared to the preoperative period $(P<.05)$. The mean BUT was similar in the dry eye group at the third month after the operation and at the preoperative period $(P>.05)$ (Table 4$)$.

\section{Discussion}

The PRK procedure damages the epithelial, nerves, and upper layers of the cornea [7]. Thus, the PRK procedure may cause various symptoms, such as pain in patients, despite the use of postoperative medications [8]. According to previous studies that predict symptoms after PRK, gender, anxiety, procedural knowledge, and spherical equivalent refractive error may preoperatively determine pain [11]. Epithelial hyperplasia after myopic laser ablation may contribute to regression in myopia. However, the relevance of epithelial hyperplasia to refractive change is not clear. While there are studies showing that myopic regression after PRK is related to the increase in corneal epithelial thickness, there are also studies that argue that it is not [17-18]. Moreover, Kang et al. [19] revealed a correlation between the increase in corneal epithelial thickness after PRK and myopic shift in controls one year after surgery. In recent literature, little is known about the effect of preoperative dry eye on post-PRK CET, OSDI scores, and 
uncorrected visual acuity. Thus, the aim of the present study was to investigate the effects of these variables. According to the results, statistically significant differences were found between the groups in terms of the CET values and OSDI scores. Thus, the null hypothesis that no differences in the post-PRK CET, OSDI scores, and uncorrected visual acuity existed between the groups was partially rejected.

Although the CET values and OSDI scores were similar in the control group at the third month after the operation and at the preoperative period, the mean OSDI score and CET value increased significantly in the dry eye group at the third month after the operation compared to the preoperative period. This study is the first to compare preoperative dry eye on the CET values, OSDI scores, and uncorrected visual acuity. Thus, a direct comparison cannot be made with the findings of previous reports. However, the postoperatively increased OSDI scores and CET values in the dry eye group can be explained by the presence of preoperative dry eye. Therefore, it can be suggested that dry eye should be treated preoperatively, followed by PRK.

Since there is no gold standard test, there are difficulties in the evaluation of dry eye [20]. To overcome these difficulties, various diagnostic tools have been developed for the evaluation of dry eye. For instance, the questionnaire method has been used frequently in previous studies for the evaluation of dry eye [16, $20-22]$. Furthermore, the OSDI has a sensitivity of $60 \%$ and a specificity of $79 \%$, and provides reliable information for dry eye in terms of severity, history, and effects [16]. Thus, in the present study, OSDI was used.

The Schirmer test alone is insufficient to determine the severity of dry eye disease. This is due to its poor repeatability [23]. A low Schirmer test value is not a contraindication for refractive laser surgery. Therefore, patients with low Schirmer test results were also included in this study.

The Schirmer test is an indicator of the aqueous component of the tear film layer. Healthy corneal epithelium and stable tear film layer are required to high-resolution vision. Tear film dysfunction can reduced tear volume and cause changes in tear composition, including elevated concentrations of inflammatory mediators and osmolarity. High osmolarity and free radicals induced by osmotic stress produce inflammatory mediators that further amplify corneal epithelium inflammation causses corneal epithelial edema.[24] We think that the increase in CET value after PRK in the dry eye group may be related to corneal epithelial edema. In our study, this level of change in corneal epithelial thickness did not affect post-prk UCVA.

Various studies have shown that the measurement of CET with spectral-domain optical coherence tomography, which measures without contact with the eye, is reliable and reproducible [25-26]. Many studies using spectral-domain optical coherence tomography have shown that CET changes after LASIK and PRK are not statistically different from each other [27, 28].

Reinstein et al. [29] measured the mean CET values in the normal corneas of healthy individuals as $53.4 \pm$ $4.6 \mu \mathrm{m}$. In the CET measurement program used in the present study, the normal value was between 50 $\mu \mathrm{m}$ and $60 \mu \mathrm{m}$. Spadea et al. [30] showed that the CET value started to increase from the first week after 
refractive laser surgery, and the thickness increase reached the highest level at approximately $6.5 \mu \mathrm{m}$ in the third month. In the present study CET measurement was performed at the third month after PRK.

In our opinion, the most important limitation of our study is that we could not clearly demonstrate whether the change in corneal epithelial thickness has an effect on the OSDI score.

\section{Conclusion}

Within the limitations of the present study, it can be concluded that increase in corneal epithelial thickness and OSDI score after PRK in the dry eye group does not affect UCVA.

Although the OSDI scores and CET values were similar in the control group at the third month after the operation and at the preoperative period, the mean OSDI score and CET value increased significantly in the dry eye group at the third month after the operation compared to the preoperative period and reduces visual comfort. Therefore, we recommend treating dry eye disease before PRK if possible. We think that it is important for dry eye patients to be adequately informed about the symptoms associated with ocular surface disease that may occur after the operation.

\section{Declarations}

\section{Funding:}

The authors declare that no funds, grants, or other support were received during the preparation of this manuscript.

\section{Competing Interests:}

The authors have no relevant financial or non-financial interests to disclose.

\section{Author Contributions:}

All authors contributed to the study conception and design. Material preparation, data collection and analysis were performed by Irfan Botan Güneş, Metin Ekinci and Hakan Öztürk. The first draft of the manuscript was written by irfan Botan Güneş and all authors commented on previous versions of the manuscript. All authors read and approved the final manuscript.

\section{References}

1. Tsubota K, Yokoi N, Watanabe $\mathrm{H}$ et al (2020) A new perspective on dry eye classification: Proposal by the Asia Dry Eye Society. Eye Contact Lens 46(1):S2

2. Dogru M, Tsubota K (2011) Pharmacotherapy of dry eye. Expert Opin Pharmacother 12(3):325-334

3. Nichols KK, Smith JA (2002) Association of clinical diagnostic tests and dry eye surveys: the NEIVFQ-25 and the OSDI. Lacrimal Gland, Tear Film, and Dry Eye Syndromes 3. Springer, pp 1177-1181 
4. McCarty CA, Bansal AK, Livingston PM et al (1998) The epidemiology of dry eye in Melbourne, Australia. Ophthalmology 105(6):1114-1119

5. Schaumberg DA, Sullivan DA, Buring JE et al (2003) Prevalence of dry eye syndrome among US women. Am J Ophthalmol 136(2):318-326

6. Seiler T, Kahle G, Kriegerowski M (1990) Excimer laser (193 nm) myopic keratomileusis in sighted and blind human eyes. In.: SLACK Incorporated Thorofare, NJ

7. Alió JL, Muftuoglu O, Ortiz D et al (2008) Ten-year follow-up of photorefractive keratectomy for myopia of more than- 6 diopters. Am J Ophthalmol 145(1):37-45. e31.

8. Garcia R, Horovitz RNC, Torricelli AAM et al (2016) Improved evaluation of postoperative pain after photorefractive keratectomy. Cornea 35(2):205-209

9. Nielsen CS, Staud R, Price DD (2009) Individual differences in pain sensitivity: measurement, causation, and consequences. The journal of pain 10(3):231-237

10. Fagerholm $P$ (2000) Wound healing after photorefractive keratectomy. Journal of Cataract \& Refractive Surgery 26(3):432-447

11. Garcia R, Torricelli AAM, Mukai A et al (2016) Predictors of early postoperative pain after photorefractive keratectomy. Cornea 35(8):1062-1068

12. Morin C, Lund JP, Villarroel T et al (2000) Differences between the sexes in post-surgical pain. Pain 85(1-2):79-85

13. Tan CO, Chong YM, Tran P et al (2015) Surgical predictors of acute postoperative pain after hip arthroscopy. BMC Anesthesiol 15(1):1-6

14. Kalkman C, Visser K, Moen J et al (2003) Preoperative prediction of severe postoperative pain. Pain 105(3):415-423

15. Garcia-Catalan M, Jerez-Olivera E, Benitez-Del-Castillo-Sanchez J (2009) Dry eye and quality of life. Archivos de la Sociedad Espanola de Oftalmologia 84(9):451-458

16. Schiffman RM, Christianson MD, Jacobsen G et al (2000) Reliability and validity of the ocular surface disease index. Arch Ophthalmol 118(5):615-621

17. Erie JC (2003) Corneal wound healing after photorefractive keratectomy: a 3-year confocal microscopy study. Trans Am Ophthalmol Soc 101:293

18. Ivarsen A, Fledelius W, Hjortdal J (2009) Three-year changes in epithelial and stromal thickness after PRK or LASIK for high myopia. Investig Ophthalmol Vis Sci 50(5):2061-2066

19. Kang DSY, Kim SW (2019) Effect of corneal cross-linking on epithelial hyperplasia and myopia regression after transepithelial photorefractive keratectomy. J Refract Surg 35(6):354-361

20. Pflugfelder SC, Geerling G, Kinoshita S Management and therapy of dry eye disease: report of the Management and Therapy Subcommittee of the International Dry Eye WorkShop et al (2007) Ocular Surface 2007;5(2):163-178

21. Dougherty BE, Nichols JJ, Nichols KK (2011) Rasch analysis of the ocular surface disease index (OSDI). Investig Ophthalmol Vis Sci 52(12):8630-8635 
22. Asiedu K, Kyei S, Mensah SN et al (2016) Ocular surface disease index (OSDI) versus the standard patient evaluation of eye dryness (SPEED): a study of a nonclinical sample. Cornea 35(2):175-180

23. Cho P, Yap M (1993 Feb) Schirmer test. II. A clinical study of its repeatability. Optom Vis Sci 70:157e159

24. Goyal S, Chauhan SK, Zhang Q, Dana R (2009) Amelioration of murine dry eye disease by topical antagonist to chemokine receptor 2. Arch Ophthalmol 2009:127:882-887. [PubMed: 19597109]

25. Sanal Doğan A, Gürdal C, Acar M (2017) Evaluation of the Effect of Topical Antiglaucoma Medications on Corneal Epithelium Thickness by Optical Coherence Tomography.GlokomKatarakt/Journal of Glaucoma-Cataract; 12(4)

26. Kim JS, Ishikawa H, Sung KR et al (2009) Retinal nerve fibre layer thickness measurement reproducibility improved with spectral domain optical coherence tomography. $\mathrm{Br} \mathrm{J}$ Ophthalmol 93(8):1057-1063

27. Rocha KM, Krueger RR (2014) Spectral-Domain Optical Coherence Tomography Epithelial and Flap Thickness Mapping in Femtosecond Laser-Assisted In Situ Keratomileusis. Am J Ophthalmol 158(2):293-301e291

28. Ma XJ, Wang L, Koch DD (2013) Repeatability of corneal epithelial thickness measurements using Fourier-domain optical coherence tomography in normal and post-LASIK eyes. Cornea 32(12):1544

29. Reinstein DZ, Archer TJ, Gobbe M et al (1995) Epithelial thickness in the normal cornea: threedimensional display with very high frequency ultrasound. Journal of refractive surgery (Thorofare, NJ: 2008;24(6):571

30. Spadea L, Fasciani R, Necozione $S$ et al (2000) Role of the corneal epithelium in refractive changes following laser in situ keratomileusis for high myopia. In.: Slack Incorporated Thorofare, NJ

\section{Tables}

Table 1. Demographic data according to the groups.

\begin{tabular}{|llll|}
\hline & Control Group & Dry Eye Group & \\
& & & \\
& $\mathrm{n}=54$ & $\mathrm{n}=52$ & P value \\
\hline Age (Mean \pm SD) & $26.35 \pm 4.05$ & $27.25 \pm 4.42$ & $=.346$ \\
\hline Gender (Male/Female) & $32 / 22$ & $28 / 24$ & $=.396$ \\
\hline
\end{tabular}

$\mathrm{SD}=$ Standard Division; $\mathrm{n}=$ number . 
Table 2. Ablation depth, uncorrected visual acuity, best corrected visual acuity levels, and the pre/postoperative Schirmer test, BUT, OSDI, central epithelial thickness, and spherical equivalent refractive error, according to the groups.

\begin{tabular}{|c|c|c|c|c|}
\hline & & $\begin{array}{l}\text { Control Group } \\
\qquad \mathrm{n}=54\end{array}$ & $\begin{array}{l}\text { Dry Eye Group } \\
\qquad n=52\end{array}$ & $P$ value \\
\hline 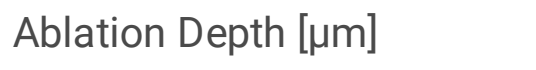 & & $46.68 \pm 1.52$ & $46.54 \pm 1.54$ & $=.598$ \\
\hline Uncorrected visual acuity * & & $0.009 \pm 0.29$ & $0.004 \pm 0.02$ & $=.370$ \\
\hline Best corrected visual acuity * & & $0.007 \pm 0.02$ & $0.009 \pm 0.03$ & $=.763$ \\
\hline \multirow[t]{2}{*}{ Schimer [mm] } & Preoperative & $23.57 \pm 5.83$ & $7.13 \pm 1.69$ & $<.001$ \\
\hline & Postoperative & $22.81 \pm 5.88$ & $6.61 \pm 1.46$ & $<.001$ \\
\hline \multirow[t]{2}{*}{ BUT [s] } & Preoperative & $14.12 \pm 2.51$ & $7.06 \pm 2.13$ & $<.001$ \\
\hline & Postoperative & $14.22 \pm 2.20$ & $6.97 \pm 1.77$ & $<.001$ \\
\hline \multirow[t]{2}{*}{ OSDI } & Preoperative & $5.00 \pm 4.25$ & $29.82 \pm 13.65$ & $<.001$ \\
\hline & Postoperative & $5.08 \pm 4.26$ & $34.93 \pm 13.25$ & $<.001$ \\
\hline Central epithelial thickness & Preoperative & $58.53 \pm 2.41$ & $59.27 \pm 2.00$ & $=.071$ \\
\hline$[\mu \mathrm{m}]$ & Postoperative & $58.59 \pm 2.81$ & $63.34 \pm 3.64$ & $<.001$ \\
\hline equivalent & Preoperative & $2.02 \pm 0.51$ & $3.32 \pm 0.59$ & $<.05$ \\
\hline \multicolumn{5}{|l|}{ refractive error [Diopter] } \\
\hline & Postoperative & $0.06 \pm 0.16$ & $0.08 \pm 0.17$ & $=.468$ \\
\hline
\end{tabular}

* Mean logMAR, s:seconds

Table 3. Control Group.

\begin{tabular}{|c|c|c|c|}
\hline & Preoperative & Three months after the operation & $P$ value \\
\hline Schirmer test [mm] & $23.57 \pm 5.83$ & $22.81 \pm 5.88$ & $<.001$ \\
\hline BUT [s] & $14.12 \pm 2.51$ & $14.22 \pm 2.20$ & $=.688$ \\
\hline OSDI & $5.00 \pm 4.25$ & $5.08 \pm 4.26$ & $=.926$ \\
\hline Central epithelial thickness $[\mu \mathrm{m}]$ & $58.53 \pm 2.41$ & $58.59 \pm 2.81$ & $=.768$ \\
\hline $\begin{array}{l}\text { Spherical equivalent refractive } \\
\text { error }\end{array}$ & $2.02 \pm 0.51$ & $0.06 \pm 0.16$ & $<.001$ \\
\hline
\end{tabular}

s: seconds 
Table 4. Dry Eye Group.

\begin{tabular}{|lllc|}
\hline & Preoperative & $\begin{array}{l}\text { Three months after the } \\
\text { operation }\end{array}$ & P value \\
\hline Schirmer test [mm] & $7.13 \pm 1.69$ & $6.61 \pm 1.46$ & $<.05$ \\
\hline BUT [s] & $7.06 \pm 2.13$ & $6.97 \pm 1.77$ & $=.697$ \\
\hline OSDI & $29.82 \pm$ & $34.93 \pm 13.25$ & $<.001$ \\
\hline Central epithelial thickness [um] & $59.27 \pm 2.00$ & $63.34 \pm 3.64$ & $<.001$ \\
\hline $\begin{array}{l}\text { Spherical equivalent refractive } \\
\text { error }\end{array}$ & $3.32 \pm 0.59$ & $0.08 \pm 0.17$ & $<.001$ \\
\hline
\end{tabular}

$s$ : seconds

Figures 


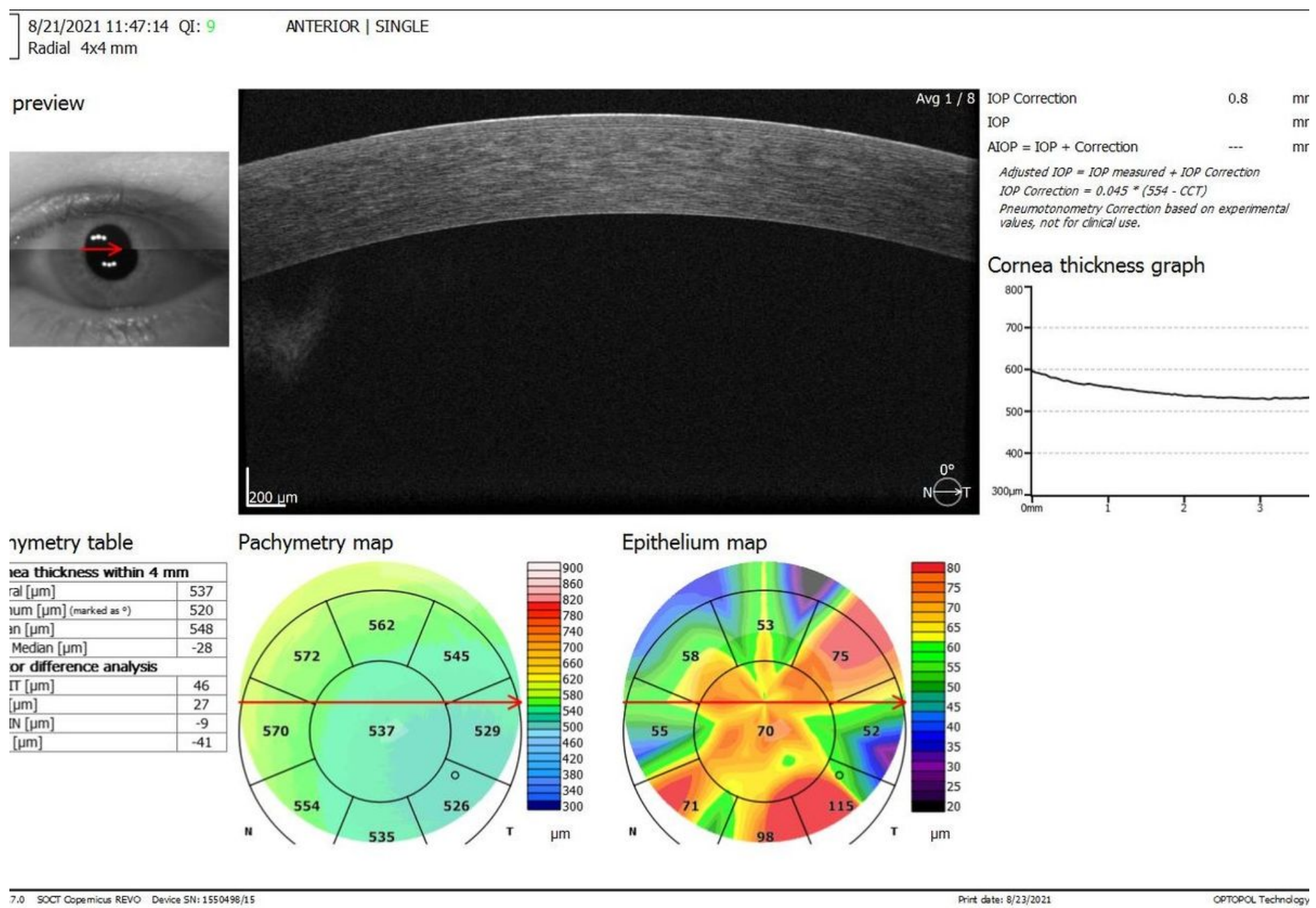

\section{Figure 1}

The corneal epithelial thickness value of the central area with a diameter of $2 \mathrm{~mm}$ was defined as the central corneal epithelial thickness. Corneal thickness measurements were performed with a Spectral Domain Optical Coherence Tomography (SD-OCT) device (Optopol Technology sp.zo. Zawiercie Polska). 